\title{
Time-dependent approach to three-body rearrangement collisions: Application to the capture of heavy negatively charged particles by hydrogen atoms
}

\author{
X. M. Tong, ${ }^{1,2, *}$ T. Shirahama, ${ }^{1}$ K. Hino, ${ }^{1,2}$ and N. Toshima ${ }^{1}$ \\ ${ }^{1}$ Institute of Materials Science, Graduate School of Pure and Applied Sciences, University of Tsukuba, \\ 1-1-1 Tennodai, Tsukuba, Ibaraki 305-8573, Japan \\ ${ }^{2}$ Center for Computational Sciences, University of Tsukuba, 1-1-1 Tennodai, Tsukuba, Ibaraki 305-8577, Japan
}

(Received 26 February 2007; published 16 May 2007)

\begin{abstract}
We present a theoretical method for Coulomb three-body rearrangement collisions solving a ChewGoldberger-type integral equation directly. The scattering boundary condition is automatically satisfied by adiabatically switching on the interaction between the projectile and hydrogen atom. Hence the outgoing wave function is obtained without the tedious procedure of adjusting the total wave function in the asymptotic region. All the dynamical information can be derived from the outgoing wave function obtained on pseudospectral grids numerically. Taking $\mu^{-}+\mathrm{H}(1 s)$ and $\bar{p}+\mathrm{H}(1 s)$ collisions as examples, we demonstrate the usefulness and powerfulness of the method and present the state-specified capture cross sections of heavy negatively charged particles by hydrogen atoms. The convergence and accuracy of the numerical procedure are examined with sufficient care.
\end{abstract}

DOI: 10.1103/PhysRevA.75.052711

PACS number(s): 34.50.Pi, 34.60. $+\mathrm{z}, 25.43 .+\mathrm{t}$

\section{INTRODUCTION}

Coulomb three-body rearrangement collisions have been studied extensively as one of the most fundamental dynamical processes. They serve as a prototype of general ion-atom collisions and electron-atom collisions. In high energy regions perturbative treatments like the Born approximation are generally applicable and the theoretical study of them is not difficult. As the collision energy decreases higher-order effects become important and we are obliged to use nonperturbative approaches. The computational time required by the nonperturbative approaches, e.g., the close-coupling method, inevitably increases dramatically. The semiclassical trajectory method, which reduces the numerical burden to some extent, can be applied for the heavy particle motion if the collision energy is not too low. In the low energy region where the de Broglie wavelengths of the relevant particles are comparable or even larger than the target geometrical size, all three particles have to be treated quantum mechanically.

In the traditional close-coupling method a timeindependent scattering equation derived from the stationary Schrödinger equation is generally solved under the boundary condition that the wave function becomes a sum of the incident plane wave and the scattered outgoing spherical wave in the asymptotic region. The scattering wave function also has to satisfy the regularity at the origin. In general, the $\mathrm{N}$-channel coupled differential equations are solved outward $N$ times using different initial conditions at the origin to obtain $N$ sets of independent solutions. Taking a linear combination of the independent solutions, one has to adjust the wave function to the proper boundary condition in the asymptotic region to obtain the $K$ matrix or the $T$ matrix. As the number of channels $N$ increases, numerical difficulties

\footnotetext{
*Corresponding author. Electronic address: tong@ims.tsukuba.ac.jp
}

increase rapidly. First, the integration of multichannel coupled equations becomes time consuming for large $N$ nearly in proportion to $N^{4}$. Second, linear independency of the $N$ sets of solutions becomes worse owing to the contamination of unphysical solutions that grow up exponentially at large distances. Requirement of a large number of channels happens when the masses of the exchanged particles differ significantly. For example, when heavy negatively charged particles like negative muons or antiprotons collide with hydrogen atoms in a $10 \mathrm{eV}$ energy region the heavy particles are mostly captured into highly excited states with the principle quantum number $n \sim \sqrt{\mu}$ extending to all the possible angular momenta $l$. Here $\mu$ is the reduced mass between the proton and the heavy particle in the unit of electron mass [1].

One way to circumvent the difficulties involving the multichannel scattering equations is to introduce the freedom of time. Although the spatial and temporal parts of the wave function can be separated for the stationary scattering problems, introducing the redundant variable of time replaces the boundary condition in space by an initial condition in the time domain. In this treatment, a wave packet [2] is prepared at a starting point to represent the incident particle and the wave packet is propagated inward following the Schrödinger equation. Though this method has a merit that it can provide an intuitive physical picture of the time evolution for the particle motion, it has a demerit that one has to sacrifice the numerical accuracy. In order to localize the wave packet within a sufficiently small region, one has to superpose many states belonging to different collision energies, and accordingly, keeping high numerical accuracy throughout the collision is difficult. In fact, Sakimoto [2-4] could not obtain the state-specified capture cross sections for antiprotons, kaons, or muons colliding with hydrogen atoms. He just presented the total capture cross sections subtracting the sum of elastic and excitation probabilities from the unity.

Esry and Sadeghpour [5] and Hesse et al. [6] calculated the state-specified capture cross sections by the hyperspherical coordinate method using a scaled mass for antiprotons. 
Scaling down the antiproton mass makes the principal quantum number of the captured states lower and the number of the coupled channels much smaller. The usage of scaled mass helps qualitative understanding of the capture process but there is no way to convert the cross sections of the scaled-mass calculations to those of the real mass quantitatively. Recently, Ovchinnikov and Macek [7] applied the advanced adiabatic approach to the problem and presented the $n$-dependent capture cross sections. Although their sophisticated method may be accurate qualitatively for the relevant process, the quantitative reliability for the minute information such as the $n$-distribution is not clear. Yamanaka and Ichimura [8] obtained the state-specified antiproton-capture cross sections solving the Faddeev equation and neglecting the angular momentum exchange between the antiproton and the electron. Even though the approximation simplifies the numerical treatment significantly, the justification of the approximation is not easy. As a matter of fact, we demonstrated in our recent study that the contribution owing to the angular momentum exchange between the projectile and the electron is important. Cohen $[9,10]$ studied the processes extensively by the classical trajectory Monte Carlo (CTMC) method. The applicability of the classical mechanics at such low energies is not clear, especially for the motion of the electron. Besides, ambiguity occurs in extracting the discrete quantum numbers $n$ and $l$ from the classical continuous quantities. To the best of our knowledge, reliable state-specified capture cross sections have not been reported yet from nonperturbative quantal calculations apart from our recent work of antiprotons colliding with hydrogen atoms [11].

\section{THEORETICAL METHOD}

\section{A. General time-dependent formalism}

The time-independent Schrödinger equation of a Coulomb three-body system in the center-of-mass frame is written as (atomic units are used hereafter unless otherwise stated)

$$
H|\Psi\rangle=E|\Psi\rangle,
$$

with

$$
H=-\frac{1}{2 \mu_{1}} \nabla_{\mathbf{r}}^{2}-\frac{1}{2 \mu_{2}} \nabla_{\mathbf{R}}^{2}+\frac{Z_{1} Z_{2}}{r}+\frac{Z_{1} Z_{3}}{\left|\mathbf{R}+a_{0} \mathbf{r}\right|}+\frac{Z_{2} Z_{3}}{\left|\mathbf{R}-a_{1} \mathbf{r}\right|} .
$$

Here $E=E_{c}+E_{1 s}$ is the total energy of the collision system in the center-of-mass frame with $E_{c}$ the incident energy of the projectile in the center-of-mass frame and $E_{1 s}$ the ground state energy of hydrogen atom. $\mathbf{r}$ and $\mathbf{R}$ are, respectively, the distances from particle 1 to particle 2 and from the centerof-mass of particles 1 and 2 to particle $3 . Z_{1}, Z_{2}, Z_{3}$ are the electric charges of particles 1,2 , and 3 , respectively. $\mu_{1}$ is the reduced mass of particles 1 and 2 , and $\mu_{2}=M_{3}\left(M_{1}\right.$ $\left.+M_{2}\right) /\left(M_{1}+M_{2}+M_{3}\right) . M_{1}, M_{2}$, and $M_{3}$ are the masses of particles 1,2, and 3, respectively, and $a_{0}=M_{2} /\left(M_{1}+M_{2}\right)$ and $a_{1}=M_{1} /\left(M_{1}+M_{2}\right)$. Circulating indices 1,2 , and 3 defines a different Jacobi set. The Hamiltonian can be recast into two forms before and after the collision for rearrangement processes as

$$
\begin{gathered}
H_{i}\left|\Psi_{i}\right\rangle=E\left|\Psi_{i}\right\rangle, \\
H_{f}\left|\Psi_{f}\right\rangle=E\left|\Psi_{f}\right\rangle,
\end{gathered}
$$

and the corresponding interactions are

$$
\begin{aligned}
& V_{i}=H-H_{i}, \\
& V_{f}=H-H_{f} .
\end{aligned}
$$

The solutions of Eq. (1) satisfying a proper boundary condition can be conveniently expressed utilizing an integral expression

$$
\begin{aligned}
\Psi^{+}(t) & =\Psi_{i}-i \int_{-\infty}^{t} e^{-i(H-E)\left(t-t^{\prime}\right)} f\left(t^{\prime}\right) V_{i} \Psi_{i} d t^{\prime} \\
& =\Psi_{i}-i \int_{-\infty}^{t} e^{i E\left(t-t^{\prime}\right)} U\left(t, t^{\prime}\right) f\left(t^{\prime}\right) V_{i} \Psi_{i} d t^{\prime}
\end{aligned}
$$

The time-dependent wave function $\Psi^{+}(t)$ satisfies Eq. (3) as $t \rightarrow-\infty$, and it also satisfies Eq. (1) at $t=0$ if $f(-\infty)=0, f(0)$ $=1$, and $f(t)$ switches on adiabatically [12], i.e., $\frac{d}{d t} f(t) \rightarrow 0$. A choice of $f(t)=\exp (-\eta|t|)$ gives a Chew-Goldberger formal solution at $t=0$ as

$$
\Psi^{+}=\Psi_{i}+\frac{1}{E-H+i \eta} V_{i} \Psi_{i},
$$

where $\eta$ is a positive infinitesimal number. We integrate Eq. (7) propagating the time-dependent part instead of solving Eq. (1) or Eq. (8). $f(t)$ can be any function so long as it is switched on adiabatically. For instance, $f(t)=\exp \left(-t^{2} / \tau^{2}\right)$ can be used for $\tau \rightarrow \infty$. Once we get $\Psi^{+}(t=0)$ by propagating Eq. (7), we can derive any dynamical information of the collision system from it.

\section{B. Numerical procedures}

We have introduced a general theory for solving scattering problems. Coulomb three-body systems cover a variety of physical processes. The characteristic of each system depends strongly on the combination of the masses and the charges of the constituting particles. We deal with heavy negatively charged particles, negative muons, and antiprotons, colliding with hydrogen atoms as examples to show how to propagate Eq. (7) effectively and accurately. For the convenience of the calculation, we choose the proton for particle 1 , the electron for particle 2 , and the negatively charged projectile for particle 3 . The initial wave function $\Psi_{i}$ of the system is the product of the ground state wave function of the hydrogen atom and the plain wave of the projectile

$$
\Psi_{i}(\mathbf{r}, \mathbf{R})=\psi_{1 s}(\mathbf{r}) e^{i \mathbf{k}_{0} \cdot \mathbf{R}},
$$

where $\mathbf{k}_{0}$ is the initial momentum of the projectile in the center-of-mass frame. The Hamiltonian of the initial channel and the corresponding interaction are

$$
H_{i}=-\frac{1}{2 \mu_{1}} \nabla_{\mathbf{r}}^{2}-\frac{1}{r}-\frac{1}{2 \mu_{2}} \nabla_{\mathbf{R}}^{2},
$$




$$
V_{i}=\frac{1}{\left|\mathbf{R}-a_{1} \mathbf{r}\right|}-\frac{1}{\left|\mathbf{R}+a_{0} \mathbf{r}\right|} .
$$

Since the total angular momentum $L$, its component $M$ onto the incident beam direction, and the parity are good quantum numbers, we can expand the time-dependent wave function as

$$
\begin{aligned}
\Psi(t) & =\sum_{L M} \Psi_{L M}(\mathbf{r}, \mathbf{R}, t) \\
& =\sum_{L M} \sum_{\alpha} F^{\alpha}(r, R, t) \Omega_{L M}^{\alpha}\left(l_{e}, l\right),
\end{aligned}
$$

with

$$
\Omega_{L M}^{\alpha}=\sum_{m_{e}, m}\left\langle l_{e} m_{e} l m \mid L M\right\rangle Y_{l_{e} m_{e}}(\hat{r}) Y_{l m}(\hat{R}) .
$$

Here $\alpha$ represents the angular momentum of the electron $l_{e}$, the angular momentum of the heavy negative particle $l$, and the parity collectively. Each $\Psi_{L M}(\mathbf{r}, \mathbf{R}, t)$ can be propagated independently. The corresponding radial part of the total Hamiltonian for a given $L M$ is written as

$$
\begin{aligned}
\mathbf{H}(r, R)= & \left\langle\Omega_{L M}^{\alpha}|H| \Omega_{L M}^{\alpha^{\prime}}\right\rangle \\
= & \left(-\frac{1}{2 \mu_{2}} \frac{\partial^{2}}{\partial R^{2}}+\frac{l(l+1)}{2 \mu_{2} R^{2}}-\frac{1}{R}\right) \delta_{\alpha, \alpha^{\prime}}+\left(-\frac{1}{2 \mu_{1}} \frac{\partial^{2}}{\partial r^{2}}\right. \\
& \left.+\frac{l_{e}\left(l_{e}+1\right)}{2 \mu_{1} r^{2}}-\frac{1}{r}\right) \delta_{\alpha, \alpha^{\prime}}+\left\langle\Omega_{L M}^{\alpha}\right| \frac{1}{R}-\frac{1}{\left|\mathbf{R}+a_{0} \mathbf{r}\right|} \\
& +\frac{1}{\left|\mathbf{R}-a_{1} \mathbf{r}\right|}\left|\Omega_{L M}^{\alpha^{\prime}}\right\rangle \\
= & \mathbf{H}^{\alpha}(R)+\mathbf{H}^{\alpha}(r)+\mathbf{V}^{\alpha, \alpha^{\prime}}(r, R) .
\end{aligned}
$$

After the partition, the time propagation from $t$ to $t+\Delta t$ can be performed by the split-operator method in the energy representation [13] as

$$
\begin{aligned}
U(t+\Delta t, t)= & e^{-i \mathbf{H}(r, R) \Delta t} \\
= & e^{-i \mathbf{V}(r, R) \Delta t / 2} e^{-i \mathbf{H}(R) \Delta t} \\
& \times e^{-i \mathbf{H}(r) \Delta t} e^{-i \mathbf{V}(r, R) \Delta t / 2}+O\left(\Delta t^{3}\right) .
\end{aligned}
$$

We first propagate the wave function a half-time step in $\mathbf{V}(r, R)$, and then a full-time step in $\mathbf{H}(r)$ and $\mathbf{H}(R)$, and finally another half-time step in $\mathbf{V}(r, R)$. The advantage of this time-propagation scheme is that each operator is diagonal in the other two dimensions. $\mathbf{V}(r, R)$ couples only different channels $\alpha \neq \alpha^{\prime}$ and $\mathbf{H}(R)$ and $\mathbf{H}(r)$ are, on the other hand, diagonal for $\left(\alpha, \alpha^{\prime}\right)$. These characters make the time propagation computationally efficient.

For three-body rearrangement collisions, we also have to deal with continuum wave functions although the key dynamics takes place mainly when the three particles are close to one another. In the direct integration method on grid points, the whole coordinate space is confined in a finite box. We have to filter out the outgoing waves at the boundary in order to eliminate the unphysical reflection. For this purpose, we add an optical potential near the boundary to absorb the outgoing particle flux. The optical potential [14] we used is

$$
V_{o p t}(\epsilon, r)= \begin{cases}0 & \text { for } r<r_{c}, \\ -i V_{0}\left(\frac{r-r_{c}}{r_{\max }-r_{c}}\right) & \text { for } r \geqslant r_{c},\end{cases}
$$

with

$$
\begin{gathered}
2 \epsilon^{1 / 2} / A<V_{0}<\epsilon^{3 / 2} A, \\
A=\sqrt{2 \mu}\left(r_{\text {max }}-r_{c}\right) .
\end{gathered}
$$

Here $\epsilon$ is the energy of the basis wave function and $r_{\text {max }}$ and $r_{c}$ are the position of the boundary in the radial direction and the position to start the filtering, respectively. $\mu$ is the reduced mass associated with coordinate $r$. We choose $r_{c}$ in such a way that $r_{c}$ is smaller for higher energy. The optical potential added to the Hamiltonian $\mathbf{H}(r)$ is

$$
V_{o p t}(r)=\sum_{i} V_{o p t}\left(\epsilon_{i}, r\right)\left|\psi_{i}\right\rangle\left\langle\psi_{i}\right| \text { for } \epsilon_{i} \geqslant 0,
$$

where $\left\{\epsilon_{i}\right\}$ and $\left\{\psi_{i}\right\}$ are the eigenenergies and eigenwave functions of $\mathbf{H}(r)$. The $V_{o p t}(r)$ depends on the grid structure but it does not depend on the collision energy. It is a universal optical potential which can be used to absorb the ejected electron in a broad energy range. Similarly we add an optical potential to $\mathbf{H}(R)$. After a long time propagation from $t=-\infty$ to $t=0$, in the interaction region, we get the stationary wave function $\Psi^{+}(t=0)$, which satisfies the proper boundary condition. Since the proton mass is much larger than the electron mass, the center-of-mass of the hydrogen atom is regarded to stay on the proton to a good approximation, and hence we set $a_{0}=0$ and $a_{1}=1$. The final wave function corresponding to the capture of the state of a principal quantum number $n$ and an angular momentum $l$ is expressed as

$$
\Psi_{f}^{\alpha}=j_{l}\left(k_{n l} r\right) \psi_{n l}(R) \Omega_{L M}^{\alpha},
$$

where $k_{n l}$ is the ejected electron momentum associated with the formation of an exotic hydrogen atom in the $n l$ state. The $T$-matrix element is given by

$$
T_{n l}^{\alpha}=\left\langle\Psi_{f}^{\alpha}\left|V_{f}\right| \Psi_{L M}^{+}\right\rangle,
$$

and the state-specified capture cross section is readily obtained as

$$
\sigma_{n l}^{\alpha}(L)=\frac{4 \mu_{2} \mu_{1} k_{n l}}{k_{0}} \sum_{\alpha}\left|T_{n l}^{\alpha}\right|^{2} .
$$

We introduce two other ways to calculate the capture cross sections. The wave function $\Psi_{L M}^{+}(0)$ can be further recast into

$$
\begin{aligned}
\left\langle\Omega_{L M}^{\alpha} \mid \Psi_{L M}^{+}\right\rangle= & \left\langle\Omega_{L M}^{\alpha} \mid \Psi_{i}\right\rangle+\sum a_{n l_{e}}^{\alpha} \psi_{n l}(r) F^{\alpha}(R) \\
& +\sum_{n l} b_{n l}^{\alpha} \psi_{n l}(R) f_{n l}^{\alpha}(r) .
\end{aligned}
$$

The first term represents the incident channel, the second term stands for the excitation and elastic channel, and the third term is the capture channel. The asymptotic form of the ejected electron wave function is 


$$
f_{n l}^{\alpha}(r)=\frac{e^{i k_{n l} r}}{r} \quad \text { for } r \rightarrow \infty .
$$

Define

$$
G_{n l}^{\alpha}(r)=\int \psi_{n l}^{*}(R)\left\langle\Omega_{L M}^{\alpha} \mid \Psi^{+}\right\rangle d R,
$$

and we obtain $b_{n l}^{\alpha}$ as

$$
\frac{k_{n l}}{\mu_{1}}\left|b_{n l}^{\alpha}\right|^{2}=\operatorname{Re}\left\{-\frac{i}{\mu_{1}}\left(r G_{n l}^{\alpha}(r)\right)^{*} \frac{\partial}{\partial r}\left(r G_{n l}^{\alpha}(r)\right)\right\}_{r_{c}},
$$

where Re denotes the real part. The definition of the capture cross sections gives

$$
\sigma_{n l}^{\alpha}(L)=\frac{\mu_{2} k_{n l}}{\mu_{1} k_{0}} \sum_{\alpha}\left|b_{n l}^{\alpha}\right|^{2} .
$$

Suppose that we calculate $b_{n l}^{\alpha}$ in Eq. (26) at each time $t$ and propagate the wave function of Eq. (7) with $f(t)$ $=\exp \left(-t^{2} / \tau^{2}\right)$ from $-\infty$ to $\infty$, then the averaged ejected electron current is

$$
\frac{k_{n l}}{\mu_{1}}\left|\bar{b}_{n l}^{\alpha}\right|^{2}=\frac{\sqrt{2}}{\sqrt{\pi} \tau} \int_{-\infty}^{\infty} \frac{k_{n l}}{\mu_{1}}\left|b_{n l}^{\alpha}(t)\right|^{2} d t .
$$

The capture cross section can be calculated in the third way as

$$
\sigma_{n l}^{\alpha}(L)=\frac{\mu_{2} k_{n l}}{\mu_{1} k_{0}} \sum_{\alpha}\left|\bar{b}_{n l}^{\alpha}\right|^{2} .
$$

Equation (29) corresponds to the procedure that we collect all the ejected electrons associated with capture to $n l$ states in the whole collision process. If the wave function $\Psi^{+}(0)$ is converged satisfactorily, the three expressions, Eqs. (22), (27), and (29), should give the same results. In the next section we examine the numerical convergence by comparing the results from these three methods.

\section{CONVERGENCE CHECK}

We adopt $f(t)=\exp \left(-t^{2} / \tau^{2}\right)$ for the switching function. In the calculations, we cannot propagate the time-dependent wave function taking the limits $\tau \rightarrow \infty$ and $\Delta t \rightarrow 0$. We have to investigate how to choose proper $\tau$ and $\Delta t$ beforehand. The switching parameter $\tau$ is regulated by the slowest motion in the system and the time step $\Delta t$ is regulated by the fastest motion on the contrary. Generally speaking, the fastest motion is the motion of the captured $1 s$ state of the exotic atom and the slowest motion is the motion of the free projectile with the incident velocity $v_{0}$. Therefore, we choose $\tau$ and $\Delta t$ taking $1 / v_{0}$ and $1 /\left|\epsilon_{1 s}^{h}\right|$ as the units, respectively, where $\epsilon_{1 s}^{h}$ is the ground-state energy of the formed exotic hydrogen atom. In the following discussions, we assume that a negative muon is the projectile. For the convenience of later discussions, we name Eq. (22) as $T$ method, Eq. (27) as C0 method, and Eq. (29) as C1 method. Table I shows the $n$-dependent capture cross sections for $L=10$ as $\sigma_{n}(L=10)=\Sigma_{\alpha, l} \sigma_{n l}^{\alpha}(L)$ calculated by the three methods. We see that the capture
TABLE I. $n$-dependent capture cross sections (in a.u.) of muons by hydrogen atoms calculated by the three methods for $L=10, E_{c}$ $=2.0 \mathrm{eV}, \tau=50$, and $\Delta t=30$.

\begin{tabular}{cccc}
\hline \hline & \multicolumn{3}{c}{$\sigma_{n}(L=10)$} \\
\cline { 2 - 4 }$n$ & $T$ & $\mathrm{C} 0$ & $\mathrm{C} 1$ \\
\hline 9 & $1.85 \times 10^{-4}$ & $1.92 \times 10^{-4}$ & $1.90 \times 10^{-4}$ \\
10 & $3.05 \times 10^{-3}$ & $3.10 \times 10^{-3}$ & $3.07 \times 10^{-3}$ \\
11 & $2.80 \times 10^{-2}$ & $2.82 \times 10^{-2}$ & $2.79 \times 10^{-2}$ \\
12 & $1.82 \times 10^{-1}$ & $1.79 \times 10^{-1}$ & $1.78 \times 10^{-1}$ \\
13 & $6.42 \times 10^{-1}$ & $6.63 \times 10^{-1}$ & $6.62 \times 10^{-1}$ \\
14 & 1.12 & 1.12 & 1.15 \\
\hline \hline
\end{tabular}

cross sections calculated by the three methods are in good agreement within a few percent difference. This comparison convinces us that the present theoretical method is reliable and the calculations are well converged.

Although the three methods give the same results after a long time propagation, the discrepancy increases as we reduce the propagation time as shown in Fig. 1. As we see from the equations, the $T$ method is more sensitive to the convergence of the wave function in the inner region while the $\mathrm{C} 0$ and $\mathrm{C} 1$ methods are sensitive to the accuracy of the asymptotic wave function at $r_{c}$. The convergence of the total wave function at a different location differs as a function of the adiabatic parameter $\tau$. For the present example, the $T$ method converges faster than the other two and the $\mathrm{C} 0$ method converges most slowly. In Eq. (7), the initial wave function multiplied by the interaction plays a role of a source term, which is localized in the inner region. If $\tau$ is too short, the negative muon and the electron cannot reach the asymptotic region at $t=0$. The electron observed in the asymptotic region at $t=0$ is produced in advance by a time required for the propagation, and the interaction $V_{i}$ has to be completely switched on. This analysis clarifies the reason why the $\mathrm{C} 0$ method converges most slowly and it approaches to the converged value from below as we increase the propagation time $\tau$. In contrast, we collect all the electrons in the asymptotic regime and the time-delay effect is smeared partly in the $\mathrm{C} 1$ method. Though the $\mathrm{C} 1$ method converges faster than the $\mathrm{C} 0$ method, it has a demerit that twice the

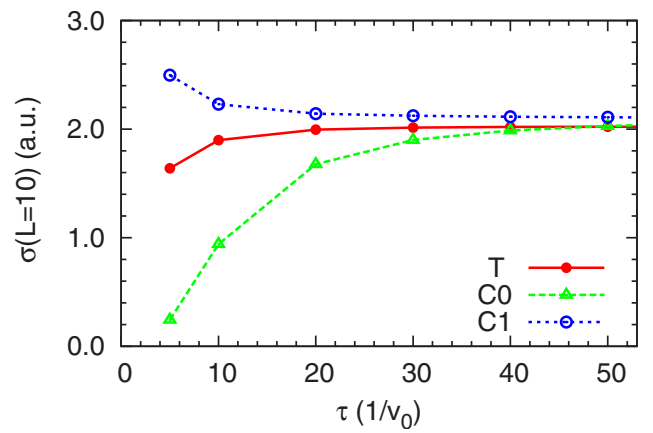

FIG. 1. (Color online) Muon-capture cross sections calculated by the three methods (see the text) as functions of the propagation time $\tau$ for $L=10$ and $E_{c}=2.0 \mathrm{eV}$. 


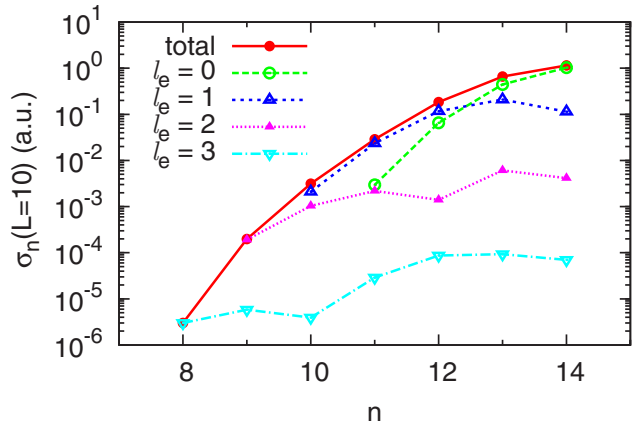

FIG. 2. (Color online) $n$-dependent muon-capture cross sections and channel contributions of different $l_{e}$ for $L=10$ and $E_{c}=2.0 \mathrm{eV}$.

propagation time is needed since the integral from $t=0$ to $t$ $=\infty$ is also to be carried out.

Next we check the convergence of the channels regarding the angular momentum of the electron. Figure 2 shows the $n$-dependence of the partial capture cross sections with respect to the final angular momentum of the electron for $L$ $=10$ and $E_{c}=2.0 \mathrm{eV}$. Ten channels are used in the range 0 $\leqslant l_{e} \leqslant 3$ for the expansion. We see that, for the large capture cross sections near $n \sim 14$, the $l_{e}=0$ channel is the dominant one and the contribution of angular momentum exchange between the muon and the electron is small. $l_{e}=1$ contributes about $10 \%$ and $l_{e}=2$ contributes less than $1 \%$. The contribution from $l_{e}=3$ is almost negligible for the major capture channels. Even though the $l_{e}=0$ channel is the dominant one for the total capture cross sections, the capture to the lower $n$ states is mainly from $l_{e}>0$ channels. For example, the $l_{e}$ $=1$ channel is the major capture channel for $n=10,11,12$ and the $l_{e}=2$ channel is the dominant one for $n=9$. Thus we can predict that, if we measure the angular distribution of the ejected electrons, the low energy electrons have isotropic distributions and the high energy electrons tend to show anisotropic distributions.

If we replace the negative muon by an antiproton, the formed protonium atoms are expected to be in even higher excited states. This increases the numerical difficulty. Although we have already presented some results for the antiproton collisions [11], we had better provide more details for the convergence check. Figure 3 depicts the ratios of the $n$-dependent antiproton-capture cross sections for different propagation time $\tau$. Similarly to the negative muon case, the

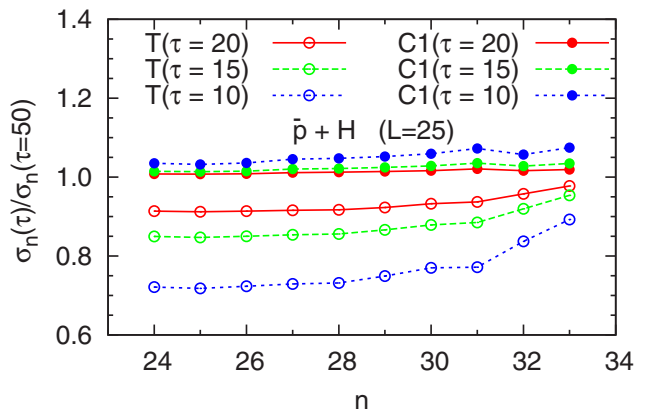

FIG. 3. (Color online) Antiproton-capture cross sections calculated by the $T$ method and the $\mathrm{C} 1$ method as functions of the propagation time $\tau$ for $L=25$ and $E_{c}=2.72 \mathrm{eV}$.

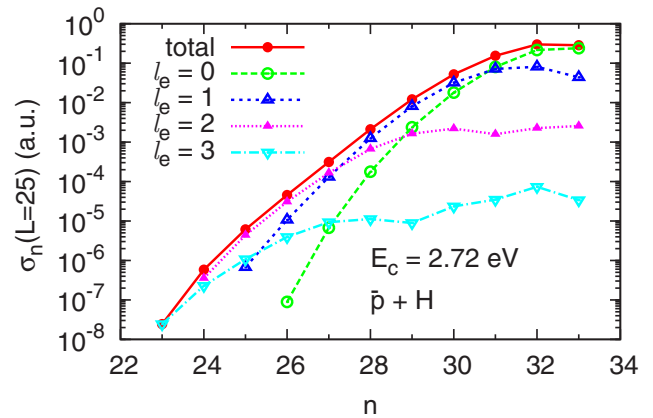

FIG. 4. (Color online) $n$-dependent antiproton-capture cross sections and the channel contributions of different $l_{e}$ for $L=25$ and $E_{c}=2.72 \mathrm{eV}$.

C0 method converges most slowly so that we do not present it in the figure. The capture cross sections also approach to the converged values from below for the $T$ method and from above for the $\mathrm{C} 1$ method consistently with the muon-capture case as shown in Fig. 1.

We also examine the distribution of the angular momenta of the ejected electron in Fig. 4. The feature of the $l_{e}$ distribution is very similar to that of the muon case. For $n \leqslant 30$, the $l_{e}=1$ channel contributes more than the $l_{e}=0$ channel, and for $n<27$, the $l_{e}=2$ channel becomes the dominant one. Similarly we can predict that, if we measure the angular distribution of the outgoing electrons, lower energy electrons have isotropic distribution while higher energy electrons tend to show anisotropic character.

We also tested the dependence of the results on $\Delta t, R_{\max }$, and $r_{\text {max }}$ to confirm that the final results are converged satisfactorily. In the present study, we always calculate the cross sections by the three methods to make sure the results are converged.

\section{RESULTS AND DISCUSSION}

At first we show the wave function $\Psi^{+}(0)$ which contains all the dynamical information of the collision system. Since it is impossible to plot a six-dimensional function $\Psi^{+}(0)$ directly, we plot the total wave function on a two-dimensional color coding $(r, R)$ by choosing $l_{e}=0$ and averaging over the angular part of $\mathbf{R}$ as shown in Fig. 5.

As a matter of fact, the plotted wave function corresponds to $\left|F^{\alpha}(r, R, t=0)\right|^{2}$ in Eq. (12) for $\alpha\left(l_{e}=0, l=L\right)$, which is the

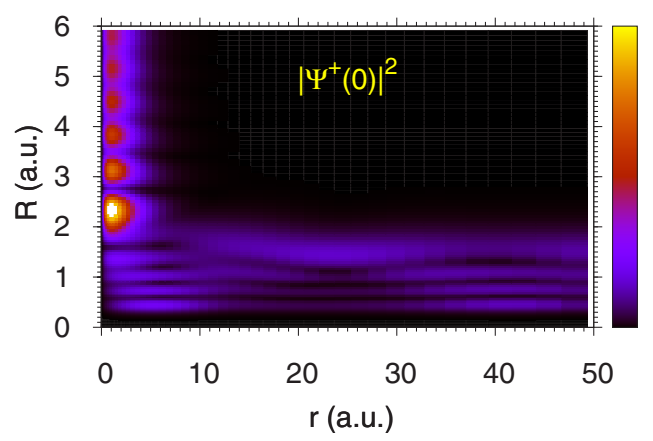

FIG. 5. (Color online) Two-dimensional plot of the total wave function $\left[\left|\Psi^{+}(0)\right|^{2}\right.$, see Eq. (7)] for $L=10, E_{c}=2.0 \mathrm{eV}$, and $l_{e}=0$. 


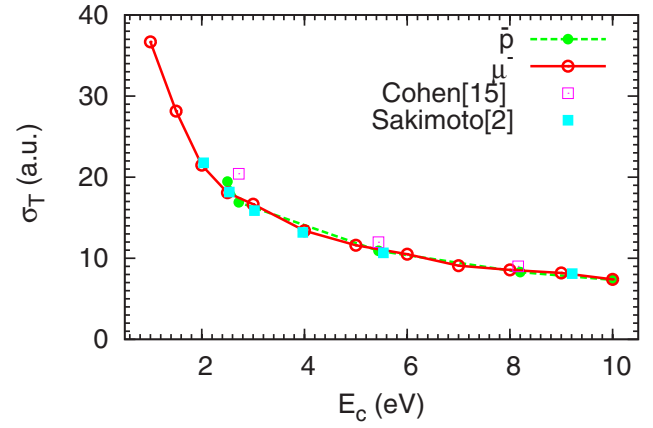

FIG. 6. (Color online) Total muon-capture cross sections (open circles) and antiproton-capture cross sections (filled circles). The total muon-capture cross sections calculated by Cohen [15] (open squares) and Sakimoto [2] (filled squares) are also plotted.

dominant channel for total capture cross sections as we have seen in Fig. 2. The electron moves to large $r$ when $R$ is small and it stands for the capture process. On the contrary, the muon moves to large $R$ when $r$ is small and it stands for the elastic scattering process. The two particles cannot move to the distant region from the proton simultaneously owing to the energy conservation. The wave function decays exponentially when both $R$ and $r$ increase simultaneously. For the muon case, we choose $R_{\max }=30-50$ a.u. and $r_{\max }$ $=150-200$ a.u. We have to use larger $r_{\max }$ to describe the motion of low energy electrons.

The total muon-capture cross sections $\sigma_{T}=\Sigma_{\alpha L, n l} \sigma_{n l}^{\alpha}(L)$ summed over the quantum numbers $n, l, L, \alpha$ are shown in Fig. 6. We also present the antiproton-capture cross sections for comparison. Besides, the muon-capture cross sections calculated by Sakimoto [4] and by Cohen [15] are presented. Our muon-capture cross sections are in good agreement with others. Although the masses of muon and antiproton differ by almost one order, the total capture cross sections are close to each other. This will raise a question of how the total capture cross sections depend on the mass of the projectile. The answer to this question may need further study.

Figure 7 shows the $L$-dependent capture cross sections $\sigma_{L}=\sum_{\alpha, n l} \sigma_{n l}^{\alpha}(L)$. In general, the capture cross sections increase almost linearly as the total angular momentum $L$ increases, reaches a peak, and then decreases as the $L$ increases further. The high- $L$ tail of the cross section drops sharply at low energies. As for higher incident energies, the peak moves

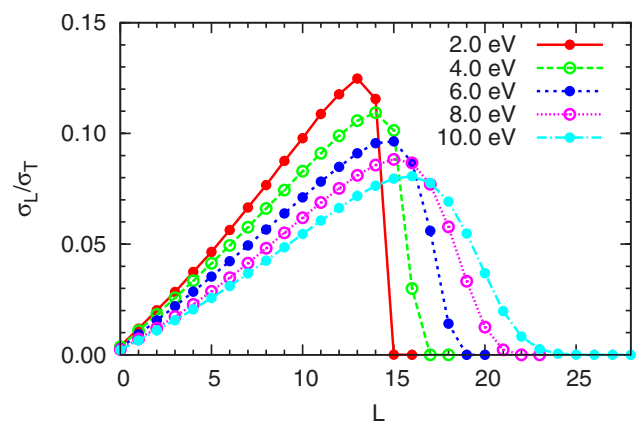

FIG. 7. (Color online) Total angular momentum $L$-dependent muon-capture cross sections normalized to the total capture cross sections at the incident energies of 2.0, 4.0, 6.0, 8.0, and $10.0 \mathrm{eV}$.

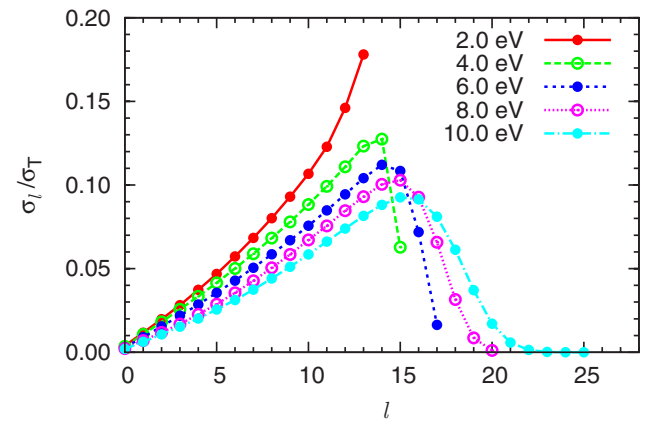

FIG. 8. (Color online) Angular momentum $l$-dependent muoncapture cross sections normalized to the total capture cross sections at the incident energies of 2.0, 4.0,6.0, 8.0, and $10.0 \mathrm{eV}$.

to higher $L$ and has a broad distribution extended to higher $L$.

The capture cross section depends on (a) the geometrical overlap of the wave functions between the formed exoticatom states and the hydrogen ground state, and (b) the penetration of the projectile wave function into the hydrogen atom. The former one does not depend on the incident energy and peaks at $n \sim \sqrt{\mu}$. Because of the conservation of the total energy, there exists an upper limit of $n$ for the capture. If the $L$ is greater than $(n-1)$, the ejected electron angular momentum $l_{e}$ must be greater than 0 . As shown in the above, the dominant capture channel is $l_{e}=0$ or $l=L$. Therefore the capture cross sections drop dramatically as $L$ increases for low incident energies. As the incident energy increases, the upper limit of $n$ increases significantly, and the capture to $l=L$ states is always possible unless the $L$ is larger than $(n-1)$. For a high incident energy, the capture cross sections should have a broad distribution peaked at $n \sim \sqrt{\mu}$ if we only consider the geometrical overlap. On the other hand, as the incident energy increases, high $L$ partial waves can penetrate into the inner region and the capture cross sections will peak at higher $L$. All these features are similar to that of the antiproton-capture case apart from that the necessary $L$ is smaller for the muon capture owing to the mass difference.

As we have seen in Figs. 2 and $4, l_{e}=0$ is the dominant channel so that the $l$-dependent capture cross sections are expected to be similar to the $L$ dependence. Figure 8 shows the $l$-dependent muon-capture cross sections $\sigma_{l}$ $=\Sigma_{\alpha L, n} \sigma_{n l}^{\alpha}(L)$. For the low energy case $\left(E_{c}=2.0 \mathrm{eV}\right)$, the highest possible $n$ is 15 and the highest $l$ is 14, which gives the maximum cross section. Owing to the existence of the upper limit of $n$ by the energy conservation, the $l$ distributions show a sharp cutoff for low incident energies. As the collision energy increases, the effect of the upper limit becomes less important and the $l$ and $L$ distributions show a similar pattern.

Figure 9 shows the $n$-dependent muon-capture cross sections $\left[\sigma_{n}=\Sigma_{\alpha L, l} \sigma_{n l}^{\alpha}(L)\right]$. Most of the muons are captured into high $n$ states above 10 . The capture cross sections decreases quickly as $n$ decreases below 10. As the collision energy increases, the $n$-dependence shows a broader distribution. All these features can be understood in a similar way as for the $L$ dependence.

Finally, we present the state-specified muon-capture cross sections $\sigma_{n l}=\Sigma_{\alpha L} \sigma_{n l}^{\alpha}(L)$ at $E_{c}=10.0 \mathrm{eV}$ in Fig. 10 . 


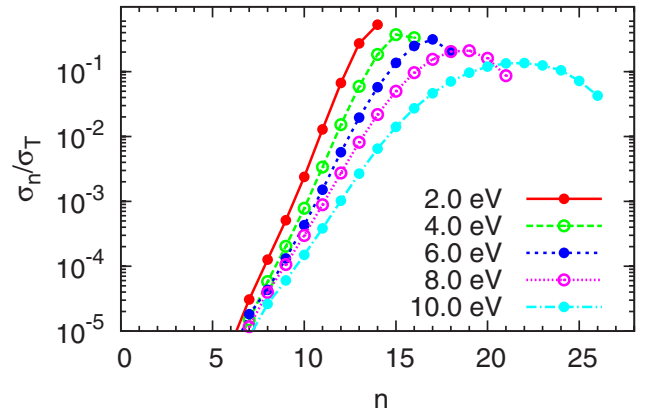

FIG. 9. (Color online) Principle quantum number $n$-dependent muon-capture cross sections normalized to the total capture cross section at the incident energies of 2.0, 4.0, 6.0, 8.0, and $10.0 \mathrm{eV}$.

The muon is mainly captured into the states with the angular momentum $l \sim 16$ and the principal quantum number $n \sim 22$. The probability of capture to the lower $n$ decreases quickly. The distribution is consistent with the $l$ and $n$ distributions in Figs. 8 and 9. Cohen [10] also predicted that the peak distribution is around $n \sim 22$ and $l \sim 15$ by the CTMC calculations.

To summarize, we have presented our recently developed theoretical method for Coulomb three-body rearrangement collisions. We transform the time-independent scattering equation to a time-dependent integral form taking account of the causality through adiabatic switching and solve the integral equation by the split-operator method in the energy rep-

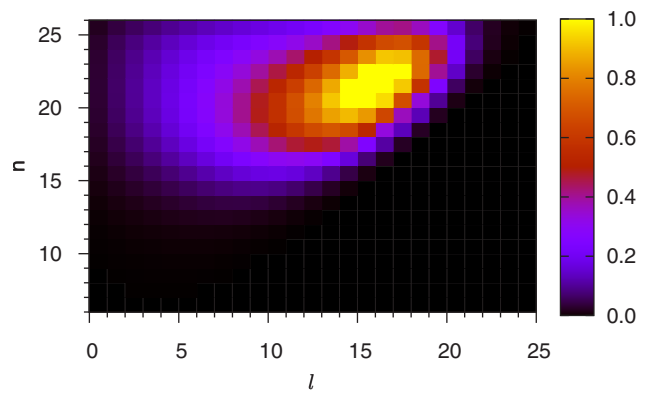

FIG. 10. (Color online) $n$ - and $l$-dependent muon-capture cross sections at $10 \mathrm{eV}$ incident energy.

resentation. The convergence has been tested by comparing the results calculated by three equivalent expressions of the scattering amplitude. The method can be extended to more general Coulomb three-body rearrangement collisions, in which the initial and final channels are described by different Jacobi sets. The combination of the masses is arbitrary, for example, such as the electron exchange in ion-atom collisions and the electron (positron) collisions with hydrogen atoms. Antisymmetrization of the wave function can be done easily for the systems which contain two identical fermions.

\section{ACKNOWLEDGMENT}

This work was supported by Grant-in-Aids for Scientific Research (B) from the Japan Society for the Promotion of Science.
[1] J. S. Cohen, Rep. Prog. Phys. 67, 1769 (2004).

[2] K. Sakimoto, Phys. Rev. A 65, 012706 (2002).

[3] K. Sakimoto, J. Phys. B 35, 997 (2002).

[4] K. Sakimoto, Phys. Rev. A 66, 032506 (2002).

[5] B. D. Esry and H. R. Sadeghpour, Phys. Rev. A 67, 012704 (2003).

[6] M. Hesse, A. T. Le, and C. D. Lin, Phys. Rev. A 69, 052712 (2004).

[7] S. Y. Ovchinnikov and J. H. Macek, Phys. Rev. A 71, 052717 (2005).
[8] N. Yamanaka and A. Ichimura, Phys. Rev. A 74, 012503 (2006).

[9] J. S. Cohen, Phys. Rev. A 36, 2024 (1987).

[10] J. S. Cohen, Phys. Rev. A 27, 167 (1983).

[11] X. M. Tong, K. Hino, and N. Toshima, Phys. Rev. Lett. 97, 243202 (2006)

[12] J. E. Avron and A. Elgart, Phys. Rev. A 58, 4300 (1998).

[13] X. M. Tong and S. I. Chu, Chem. Phys. 217, 119 (1997).

[14] J. Perie and G. Jolicard, J. Phys. B 26, 4491 (1993).

[15] J. S. Cohen, Phys. Rev. A 59, 1160 (1999). 